\title{
Depletion of PKD1 by an antisense oligodeoxynucleotide induces premature G1/S-phase transition
}

\author{
Hyunho Kim ${ }^{1}$, Yoonhee Bae ${ }^{1}$, Woocho Jeong ${ }^{1}$, Curie $\mathrm{Ahn}^{2}$ and Seongman Kang ${ }^{*, 1}$ \\ ${ }^{1}$ Graduate School of Biotechnology, Korea University, Seoul 136-701, Korea; ${ }^{2}$ College of Medicine, Seoul National \\ University, Seoul 110-744, Korea
}

\begin{abstract}
Autosomal dominant polycystic kidney disease (ADPKD) is characterized by the growth of epithelial cells and the influx of cyst fluid. The 14-kb mRNA of the polycystic kidney disease gene, PKD1, encodes the polycystin-1 protein, whose function remains unknown. In this study, we observed that polycystin-1 localized in epithelial cell-cell contacts of 293 cells. We found, by bromodeoxyuridine (BrdU) incorporation experiments and Western blot analysis of S-phase-specific cyclins, that the depletion of polycystin-1 led to an increased cell proliferation rate and caused a premature G1/S-phase transition. In addition, we showed that the depletion of polycystin-1 reduced the amount of p53 in 293 cells irradiated by UV light, suggesting that polycystin-1 acts as a regulator of $G 1$ checkpoint, which controls entry into the $S$ phase and prevents the replication of damaged DNA. Our results might provide an insight into the formation and progression of ADPKD cysts.

European Journal of Human Genetics (2004) 12, 433-440. doi:10.1038/sj.ejhg.5201136

Published online 31 March 2004
\end{abstract}

Keywords: ADPKD; PKD1; antisense oligonucleotides; p53

\section{Introduction}

Autosomal dominant polycystic kidney disease (ADPKD) is one of the most common inherited human diseases and is estimated to occur in the population with a frequency of 1 in 1000. The presence of renal cysts is the major clinical feature of the disease and cysts appear to increase in size and number throughout the lifetime of an affected individual. ${ }^{1-3}$ Although the renal lesion is the most prominent feature, ADPKD is a systemic disorder with a variety of other manifestations including liver cysts, cerebral aneurysms and cardiac valvular abnormalities. ${ }^{4,5}$

Linkage studies have revealed at least three forms of ADPKD. PKD1, which is the most common form and accounts for $85 \%$ of all cases, maps to chromosome

*Correspondence: S Kang, Graduate School of Biotechnology, Korea University, Seoul 136-701, Korea. Tel: +8223290 3448; Fax: +82 2927 9028; E-mail: skang@korea.ac.kr

Received 16 May 2003; revised 24 October 2003; accepted 30 October 2003 16p13.3. ${ }^{6-8}$ The second type, PKD2, which affects most of the remaining afflicted individuals, maps to chromosome $4 \mathrm{q} 21-23 .{ }^{9,}{ }^{10}$ A third, rare form has not yet been mapped. ${ }^{11}$,

${ }^{12}$ Even though all types of ADPKD present with an identical profile of extrarenal manifestations (including liver cysts and aneurysms), PKD1 is the most severe, with a lower median survival and a higher risk of progressing to endstage renal disease. ${ }^{13,14}$ The PKD1 gene encodes a $14 \mathrm{~kb}$ mRNA that is derived from 46 exons that extend over $\sim 50 \mathrm{~kb}$ of genomic DNA. The PKD1 gene product, polycystin-1, is 4302 amino acids in length and is likely to be an integral membrane glycoprotein that may be involved in cell-cell or cell-matrix interactions. ${ }^{15-17}$ However, the precise function of the normal polycystin-1 protein as well as the mechanism by which the mutated protein gives rise to the cystic phenotype of ADPKD remain unknown.

To understand the cellular function of polycystin- 1 and its role in pathogenesis, we have used the transfection of antisense oligodeoxynucleotides (ODNs) as our investigative approach. Properly designed antisense ODNs are 
effective inhibitors of protein synthesis, and their presence leads to lower levels of specific cellular proteins. ${ }^{18-20}$ In this approach, certain regions of a target mRNA are chosen to be annealing sites for antisense ODNs, and the resulting formation of an RNA-DNA duplex abolishes protein synthesis either by directly blocking translation or by mediating the degradation of target mRNA through RNase $\mathrm{H}$ activity. ${ }^{21}$ Here, we show, by antisense ODN-mediated depletion of polycystin-1, that polycystin-1 plays a role in the regulation of the cell cycle.

\section{Materials and methods Immunofluorescence microscopy}

Cells were grown on coverslips to $70-80 \%$ confluency in order to obtain cells in contact with other cells. ${ }^{22,23}$ Cells were fixed in $3.7 \%$ formaldehyde for $15 \mathrm{~min}$ and quenched for $10 \mathrm{~min}$ in PBS containing $50 \mathrm{mM} \mathrm{NH}_{4} \mathrm{Cl}$. Subsequently, the cells were permeabilized for $10 \mathrm{~min}$ in PBS containing $0.1 \%$ Triton $\mathrm{X}-100$. Coverslips with the cells were blocked and then reincubated for $3 \mathrm{~h}$ at room temperature with the primary antibodies (1:10). The coverslips were incubated for $1 \mathrm{~h}$ with the secondary antibodies (Jackson Laboratories, West Grove, PA, USA) each at 1:200. They were mounted on glass slides using the FluoroGuard ${ }^{\mathrm{TM}}$ Antifade Reagent (Bio-Rad Laboratories, Hercules, CA, USA).

\section{Immunoprecipitation}

Cells were grown to 70-90\% confluency. Cells were lyzed in PBS buffer containing $0.5 \%$ NP-40. Lysates were precleared by incubation with protein A-Sepharose (Pharmacia Biotech) for $1 \mathrm{~h}$ and incubated with $1 \mu \mathrm{g}$ of PKD1 (C20) antibody (Santa Cruz Biotechnology). After incubation overnight at $4^{\circ} \mathrm{C}$ with rotation, protein A-Sepharose (Pharmacia Biotech) was added, and the reaction mixture was incubated for $2 \mathrm{~h}$ at $4{ }^{\circ} \mathrm{C}$ with rotation. The beads were washed with PBS. Bound proteins were separated on a $4 \%$ SDS-PAGE gel.

\section{Antisense ODNs}

The phosphorothioated ODNs, which correspond to nucleotides 1-20 of the translation initiation site of the PKD1 mRNA (Figure 3a), were synthesized by solid-phase phosphoramidite chemistry. For ODN treatment of cells, LipofectAMINE (GIBCO, USA) and $2.5 \mu \mathrm{M}$ of the antisense ODN (or sense ODN) were added. The amount of ODN was determined by our previous several experiments and other known reports. ${ }^{19,20}$

\section{Determination of growth kinetics}

To determine the growth kinetics of 293 and MDCK cells cultured in DMEM with serum, we plated $5 \times 10^{5}$ cells in $60 \mathrm{~mm}$ dishes and counted cell numbers after 24,48 and $72 \mathrm{~h}$.

\section{Incorporation and detection of BrdU}

293 and MDCK cells were serum-starved for $48 \mathrm{~h}$ and then transfected either with the ODN/LipofectAMINE complex or LipofectAMINE only. After a $5 \mathrm{~h}$ incubation, DMEM containing $20 \mu \mathrm{BrdU}$ and 8\% FBS were added and cells were incubated for $13 \mathrm{~h}$. To fix cells, we added $4 \%$ formaldehyde. After a 10-min incubation, the cells were washed, followed by the addition of $2 \mathrm{~N} \mathrm{HCl}$ for denaturing DNA. This mixture was incubated for $1 \mathrm{~h}$. As the primary antibody, the anti-BrdU (1:100) antibody (Zymed, USA) was added. Cells were incubated with the secondary antibody, fluorescein isothiocyanate (FITC)-conjugated secondary antibody (Jackson Laboratories, West Grove, PA, USA). The cell nuclei were then labeled with $1 \mu \mathrm{g} / \mathrm{ml}$ of 4',6-diamidino-2-phenylindole (DAPI) for $5 \mathrm{~min}$.

\section{Western blot analysis}

For Western blot analysis, proteins were separated on a $12 \%$ SDS-PAGE gel and transferred onto nitrocellulose membranes. Membranes were blocked with 5\% nonfat dry milk for $1 \mathrm{~h}$ and then incubated with primary antibodies. The antibodies are described in figure legends. After washing, membranes were incubated for $1 \mathrm{~h}$ at room temperature with peroxidase-labeled secondary antibody (Santa Cruz Biotechnology). The membrane-bound antibodies were detected by the enhanced chemiluminescence (ECL) detection system (Amersham Bioscience).

\section{UV irradiation}

UV irradiation was carried out at room temperature using a germicidal UV light (chiefly $254 \mathrm{~nm}$ ). The fluence rate of the UV light source, which was measured prior to each
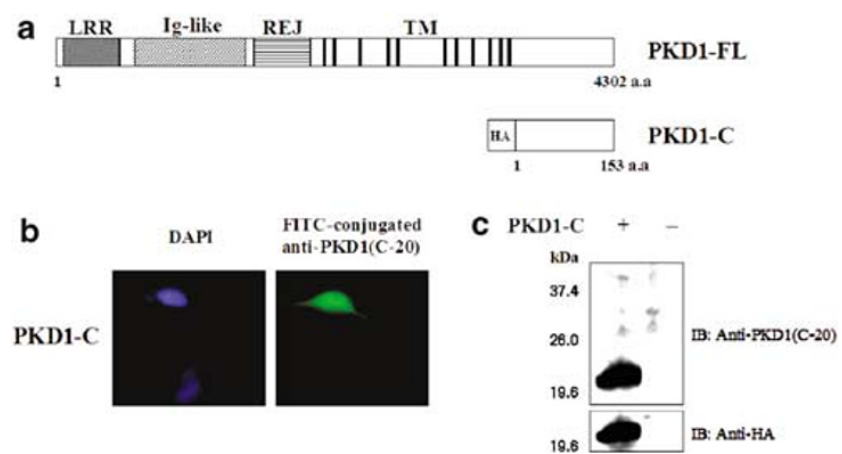

Figure 1 (a) Schematic structure of expressed recombinant polycystin-1 constructs: PKD1-FL, full-length polycystin-1 molecule, and PKD1-C, HA-tagged polycystin-1 C-terminal region. Leucine-rich repeat (LRR), Ig-like repeat (lg-like), REJ domain (REJ) and transmembrane region (TM) are indicated. (b) Immunofluorescence staining using PKD1 C-terminal antibody, goat polyclonal anti-PKD1 (C-20: sc-10372, Santa Cruz Biotechnology) of 293 cells transfected with PKD1-C. The cell nuclei were stained by DAPI. (c) Western blot of 293 cells transfected with PKD1-C, the C-terminal region of recombinant polycystin- 1 . Western blot of anti-HA (Y-11: sc-805, Santa Cruz Biotechnology) and anti-PKD1 (C-20) are identical, indicating that PKD1 (C-20) is specific to PKD1. 
experiment with a UVX radiometer, was about $80 \mathrm{~J} / \mathrm{m}^{2}$ / min. Cells were irradiated in the absence of medium, whereupon fresh medium was added and the cells were immediately reincubated at $37^{\circ} \mathrm{C}$.

\section{Results}

Endogenous PKD1 product, polycystin-1, localizes to cell-cell contacts

The characterization of specific antibodies against the potentially multifunctional protein polycystin-1 is a crucial prerequisite for addressing its role. For cellular localization and function analysis of endogenous polycystin-1 in epithelial cells, we used a C-terminal antibody, PKD1 (C-20; sc-10372). We characterized the specificity of this antibody using a recombinant polycystin-1. As shown in Figure 1a, a truncation including C-terminal region (PKD1-C), which was tagged with HA, was used for transient expression in 293 cells. The truncated PKD1 construct, PKD1-C, was expressed and revealed by immunofluorescence and Western analysis using PKD1 (C-20) antibody (Figure $1 \mathrm{~b}$ and $\mathrm{c}$ ): the Western blot of anti-HA was identical to that of anti-PKD1 (C-20). Thus, the PKD1 Cterminal antibody, PKD1 (C-20), is specific to PKD1 and available to study PKD1.

We used 293 cells, human epithelial kidney cells, to study the localization and function of polycystin-1. To address the subcellular localization of endogenous polycystin-1 in 293 cells, we performed immunostaining of polycystin-1 with the PKD1 (C-20) antibody. 293 cells showed primarily membrane staining at sites of cell-cell
A

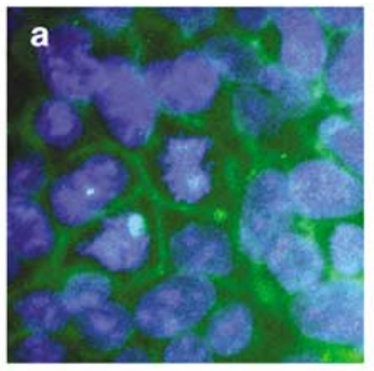

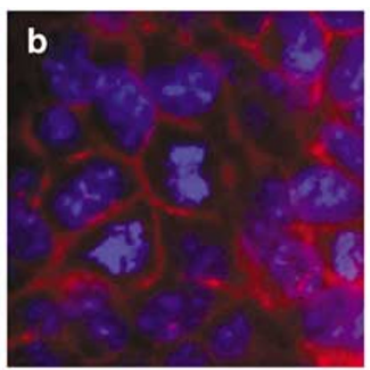

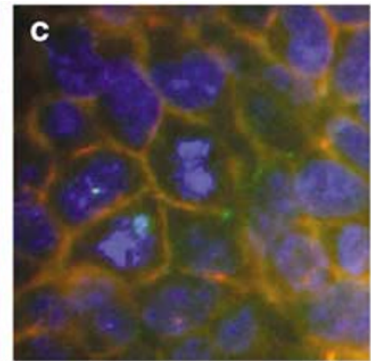

B

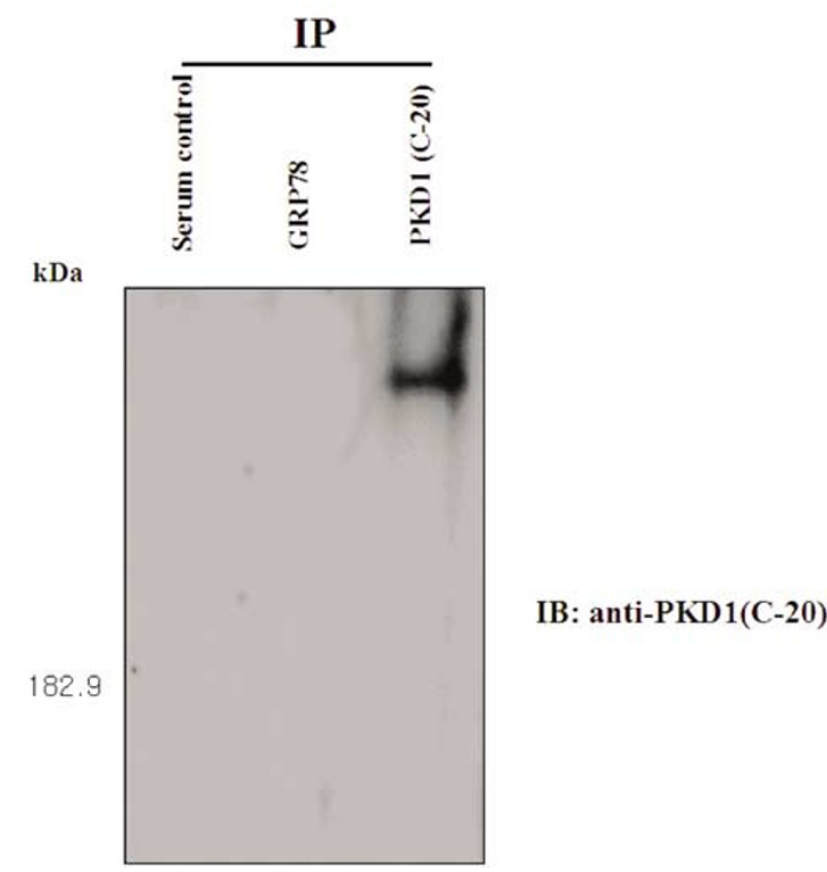

Figure 2 Subcellular localization of polycystin-1 in 293 cells. (A) Immunofluorescence staining with antibody, anti-PKD1 (C-20) (a), anti- $\beta$-catenin antibody (sc-7199; Santa Cruz Biotechnology) (b), and superimposition of PKD1 antibody and $\beta$-catenin antibody (c). Anti- $\beta$-catenin antibody, which is localized in the membrane, was used as a localization control. (B) Immunoprecipitation of goat preimmune serum, irrelevant goat polyclonal antibody, GRP 78 (sc-1050; Santa Cruz Biotechnology) and goat polyclonal PKD1 (C-20) in 4\% SDS-PAGE. The position of the molecular weight (kDa) is shown on the left. Serum control; goat preimmune serum. 
contact and some weak intracellular staining was shown (Figure 2a), as shown in other reports. ${ }^{25-27}$ No staining was observed with the secondary antibody alone (data not shown). It can be noted that no signal was detected at the isolated cells and the cell-free borders. We have also examined endogenous polycystin-1 expression by immunoprecipitation with PKD1 (C-20). We observed an $\sim 400 \mathrm{kDa}$ single band in $4 \%$ SDS-PAGE (Figure $2 \mathrm{~b}$ ). In addition, MDCK cells, canine-derived epithelial cells, were investigated and the same results were shown as in 293 cells (data not shown).

\section{PKD1 antisense ODN causes cell proliferation}

Since the significant feature of ADPKD is epithelial cell growth in the kidneys, we speculated that polycystin-1 might regulate the cell cycle. To prove this presumption, we inhibited PKD1 expression by phosphorothioated antisense oligonucleotides (ODNs) to simulate to the pathogenesis of ADPKD. As previously mentioned by other reports, ${ }^{19,20}$ antisense ODNs that are directed to the region of translation initiation on the corresponding mRNA have optimal activity, so we synthesized 20 mer-PKD1 sense ODN and PKD1 antisense ODN at the translation initiation site of PKD1 (Figure 3a). We determined the cellular levels of the polycystin- 1 protein to assess whether the transfection of 293 and MDCK cells with the PKD1 antisense ODN reduces the amount of polycystin-1 proteins. Following transfections, we prepared cell lysates after $24 \mathrm{~h}$ incubation. Proteins extracts were separated by $4 \%$ SDS-PAGE for Western blotting. As expected, the presence of PKD1 antisense ODN substantially reduced the expression of polycystin-1 in transfected 293 cells and MDCK cells (Figure 3b).

We compared the proliferation rates of 293 and MDCK cells that had been transfected with the PKD1 antisense ODN with the growth rate of cells that had been transfected with no ODN or the PKD1 sense ODN. In DMEM containing 8\% FBS, 293 and MDCK cells transfected with the PKD1 antisense ODN proliferated more quickly than cells that had been transfected with no ODN or the PKD1 sense ODN (Figure $4 \mathrm{a}$ and b). Our immunofluorescence experiments did not show cells with apoptotic features, indicating that the increased cell numbers were affected by increased cell proliferation, not decreased apoptosis (data not shown). Taken together, we conclude that the function of PKD1 might be involved in regulating cell proliferation.

PKD1-depleted 293 and MDCK cells exit G1 early To investigate whether the increased growth rate of 293 and MDCK cells by the PKD1 antisense ODN is associated with early entry into the $S$ phase in the cell cycle, we
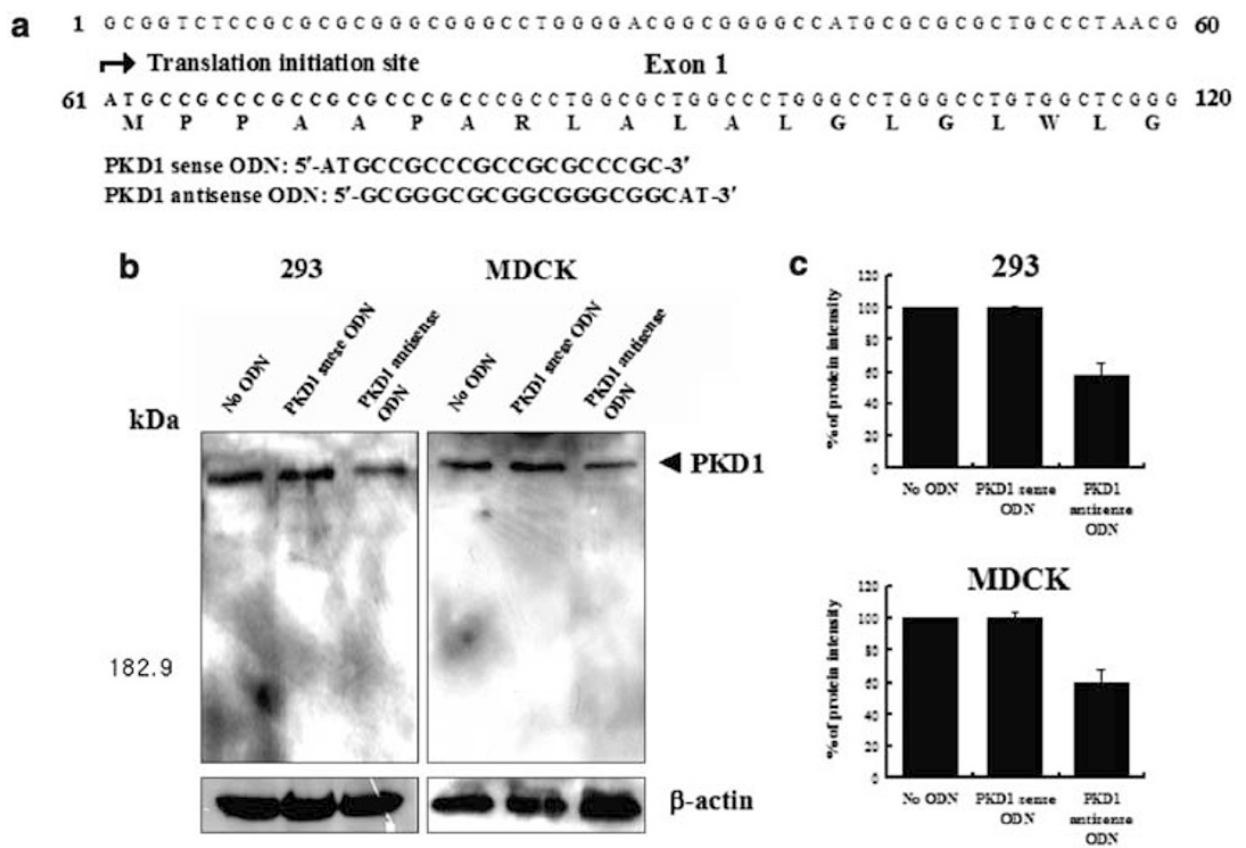

Figure 3 (a) Sequences of ODN for the antisense experiments and their target region of PKD1. Out of the first 20 base pairs of the translated region, 19 are identical between humans and dog, as shown by the GenBank sequence for dog that contains the complete coding sequence (AY102170) (b) Western blot analysis for polycystin-1 of 293 and MDCK cells lysate in 4\% SDS-PAGE. 293 and MDCK cells transfected with no ODN, PKD1 sense ODN and PKD1 antisense ODN were used for the Western blot analysis with anti-PKD1 (C-20). (c) The level of PKD1 was quantified with TINA2.0. ${ }^{24}$ Experiments were repeated three times and similar results were obtained. 
a

293

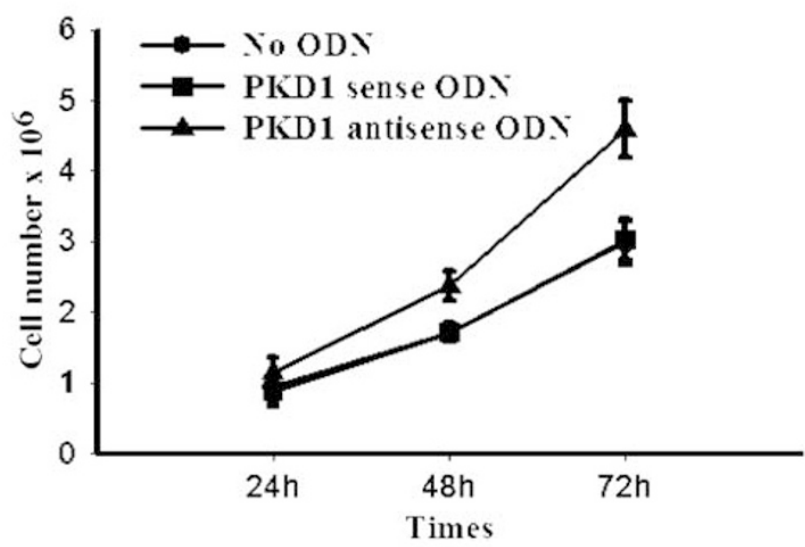

b

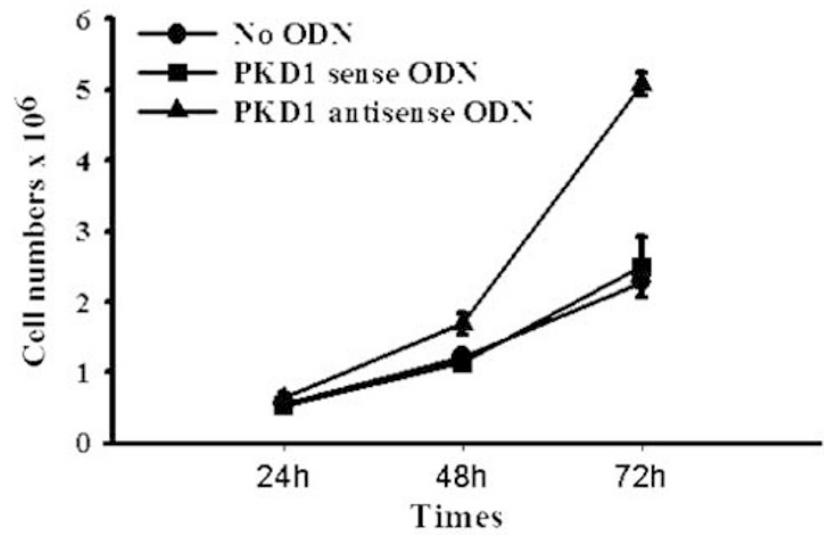

Figure 4 Growth kinetics of 293 and MDCK cells transfected with the PKD1 sense or antisense ODNs. (a, b) 293 and MDCK cells were cultured in DMEM containing $8 \% \mathrm{FBS}$. The data represent the means of three experiments carried out.

carried out BrdU pulse labeling experiments over a given period of time (Figure 5), since 5-bromo-2'-deoxyuridine (BrdU), a thymidine analog, can selectively incorporate into cellular DNA during the $S$ phase. As shown in Figure 5a, 293 and MDCK cells were synchronized into the $\mathrm{G} 1$ phase by $48 \mathrm{~h}$ serum starvation. PKD1 sense and antisense ODN were then transfected into 293 and MDCK cells and the cells were cultured in the presence of BrdU over a given period of time.

The pulse labeling experiments showed that the cells transfected with the PKD1 antisense ODN were labeled with BrdU over a given period of time $(13 \mathrm{~h})$, whereas 293 and MDCK cells transfected with no ODN or with the PKD1 sense ODN as controls were not labeled (Figure 5b). After $18 \mathrm{~h}$, however, 293 and MDCK cells in DMEM containing $8 \%$ FBS transfected with no ODN or with the
PKD1 sense ODN also were labeled (data not shown). These results imply that PKD1 antisense ODN-transfected 293 and MDCK cells enter the S phase of the cell cycle early, when compared to no ODNs or PKD1 sense ODNtransfected cells. Thus, a reduction of polycystin-1 proteins by the PKD1 antisense ODNs induces an early G1 exist and polycystin-1 may play a critical role in the G1/S-phase transition of the cell cycle.

Cells depleted of PKD1 show elevated levels of cyclin A Cyclins and cyclin-dependent kinases (CDKs) are key regulators of the eukaryotic cell cycle. In mammalian cells, different cyclin-CDK complexes are involved in regulating different cell cycle transitions. Cyclin A participates in S-phase progression. To determine whether early G1 exists in polycystin-1-depleted cells is associated with the induced expression of S-phase-specific proteins, we investigated amounts of cyclin A, and kinase CDK2 in 293 and MDCK cells that had been transfected with no ODN, the PKD1 sense or PKD1 antisense ODNs. In DMEM containing 8\% FBS, a significant increase of cyclin A proteins was observed $10-12 \mathrm{~h}$ after transfection in 293 and MDCK cells that had been transfected with the PKD1 antisense ODN (Figure 6). The levels of the CDK2 protein showed no change, regardless of the presence of ODNs. These results show that depletion of polycystin-1 in 293 and MDCK cells that had been transfected with the PKD1 antisense ODN leads to an early exit from the G1 phase, since elevated levels of cyclin A indicate that cells exit the G1 phase.

Cells depleted of PKD1 decrease p53 in UV irradiation Cells are constantly exposed to both endogenous and exogenous genotoxic agents including UV light that damages DNA and threatens their genomic stability. To repair damaged DNAs efficiently and hence protect genetic information, growing cells transiently arrest their growth. The product of the tumor suppressor p53 gene is a major component of the cellular response to DNA damage in mammalian cells. ${ }^{28}$ Thus, unrepaired DNA damage in UVirradiated cells induces an accumulation of p53 and subsequently G1-phase cell cycle arrests.

We speculated that a loss of function of PKD1 might cause premature G1 exit, although unrepaired DNA still remains. To investigate the amount of p53 after UV irradiation, various doses of UV were used to irradiate 293 cells and the expression of p53 was analyzed 4, 8 and $12 \mathrm{~h}$ after the irradiation (Figure $7 \mathrm{a}$ ). Immunoblot analyses showed that the level of p53 was increased in a UV doseand time-dependent manner: the higher the irradiation dose, the more and longer p53 was expressed.

Since p53 stabilizes to halt proliferation and to allow DNA repair, the amount of p53 is expected to decrease in cells transfected with the PKD1 antisense ODN. To test this hypothesis, 293 cells were incubated for $16 \mathrm{~h}$ after 
a

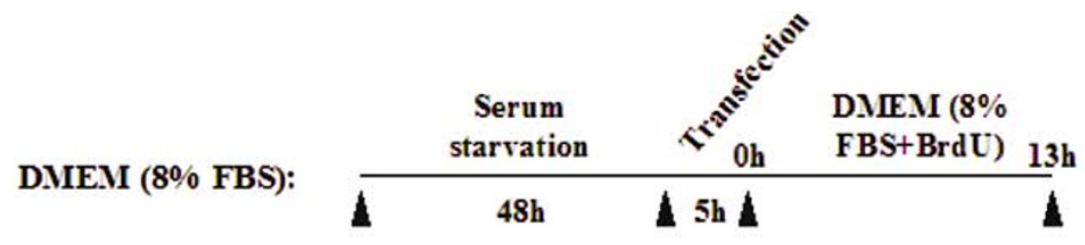

b

FITC-conjugated anti-BrdU

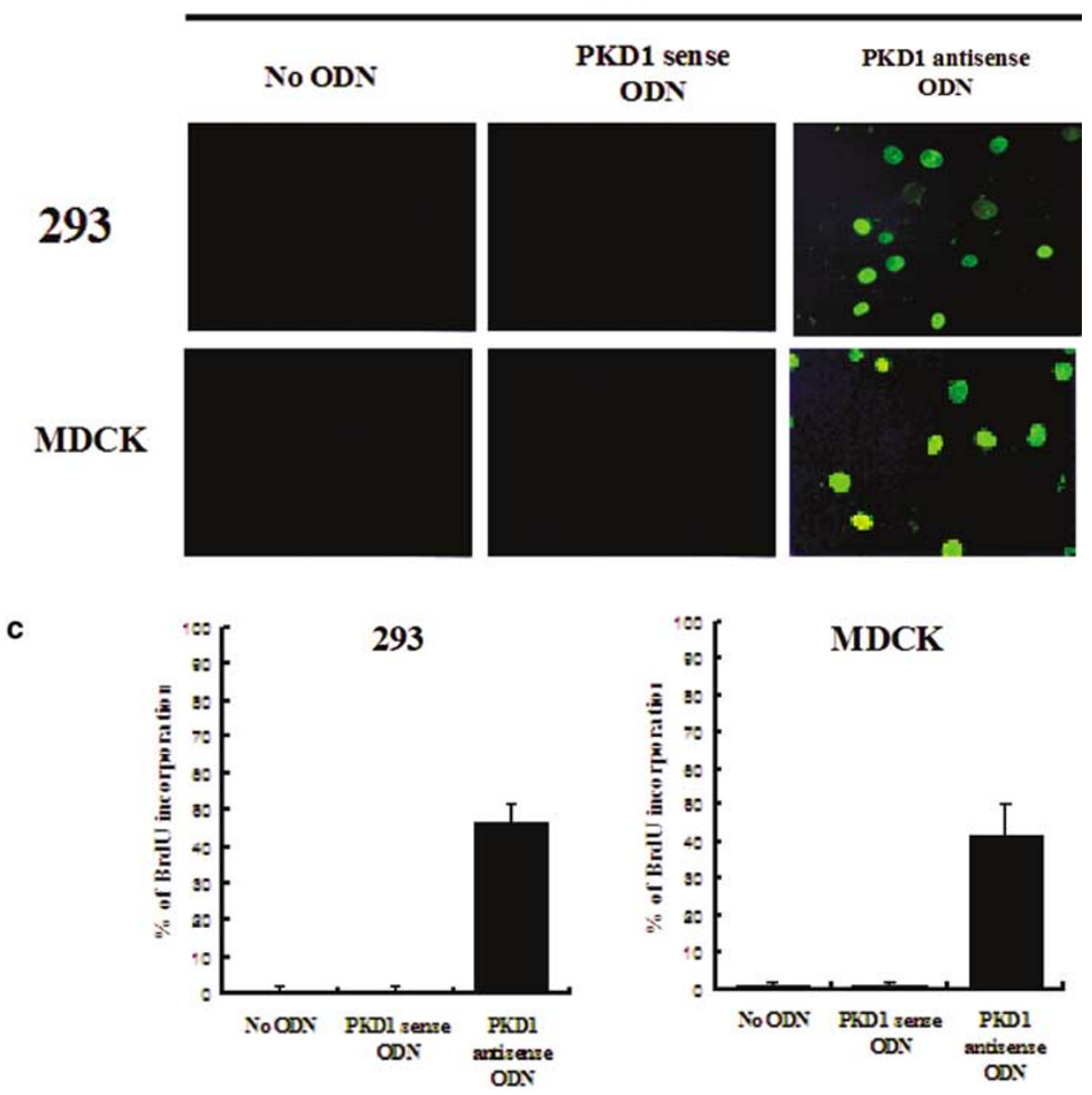

Figure 5 Effects of polycystin-1 on the cell cycle were determined by BrdU staining of 293 and MDCK cells that had been transfected with the PKD1 sense or PKD1 antisense ODNs. (a) The schematic representation of the time course used for transfection of 293 and MDCK cells with the PKD1 sense or PKD1 antisense ODNs. (b) 293 and MDCK cells were cultured in DMEM containing BrdU and $8 \%$ FBS. The cells were then stained with FITC-conjugated anti-BrdU antibody. (c) The percentage of BrdU incorporation in 293 and MDCK cells was quantitated.

transfected with no ODN or ODNs and exposed by a UV dose of $16 \mathrm{~J} / \mathrm{m}^{2}$, and p53 was then determined (Figure $7 \mathrm{~b}$ ). As expected, the level of p53 in 293 cells transfected with the PKD1 antisense ODN decreased about $60 \%$ more than no ODN and PKD1 sense ODN at $12 \mathrm{~h}$ after UV irradiation. This result was similarly shown in MDCK cells (Figure 7c). This result suggests that the loss of function of PKD1 causes a decrease of p53 that is followed by premature G1/S-phase transition.

\section{Discussion}

In this study, we used PKD1 C-terminal antibody, PKD1 (C20 ). We characterized the antibody more by immunofluorescence and immunoprecipitation experiments (Figures 1 and 2) and had the confidence of the antibody. We showed that PKD1 product, polycstin-1, localized in the cell-cell contacts of 293 using PKD1 antibody, PKD1 (C-20). This result is in agreement with the previous experiments described by others. ${ }^{25-27}$ 


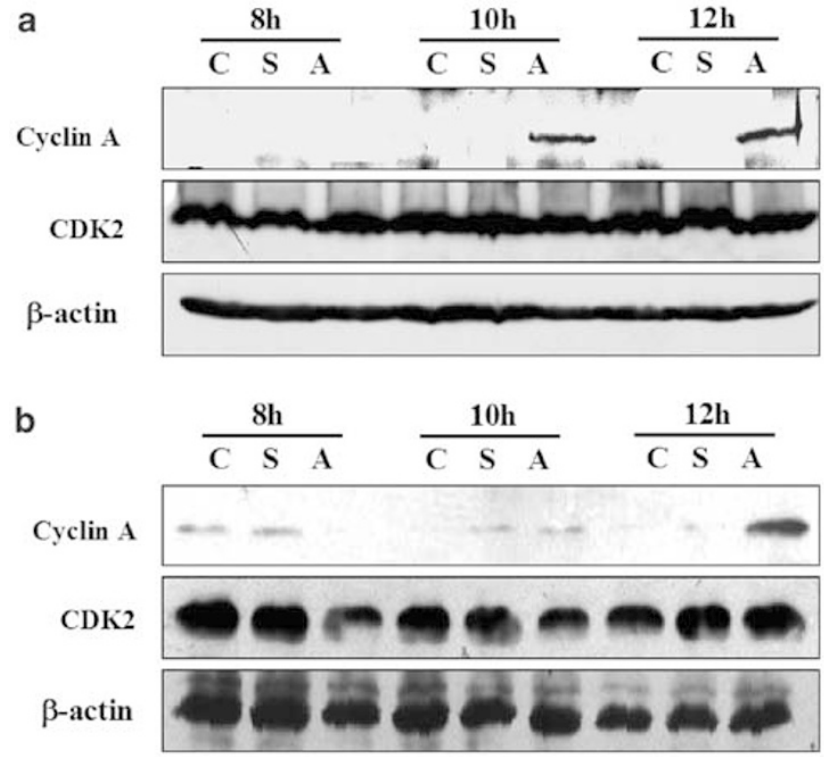

Figure 6 Analysis of cyclin A and CDK2 expression in 293 and MDCK cells. Western blot showing levels of cyclin A (sc-751 antibody; Santa Cruz Biotechnology) and CDK2 (sc-163 antibody; Santa Cruz Biotechnology) in cells transfected with ODNs. (a) In 293 cells, a significant expression of cyclin A proteins was observed 10-12 h after transfection. (b) In MDCK cells, a significant expression of cyclin A proteins was observed $12 \mathrm{~h}$ after transfection. C; No ODN, S; PKD1 sense ODN, A; PKD1 antisense ODN.

The formation and progression of ADPKD cysts are caused by the proliferation of epithelial cells. This phenomenon implicates that polycystin-1 might be associated with the cell cycle. In this study, we show that the depletion of polycystin-1 in 293 and MDCK cells by use of the PKD1 antisense ODNs results in an increased rate of cell proliferation. The depletion of PKD1 causes cells to enter the $S$ phase early and to synthesize DNA, as shown by the BrdU incorporation experiments. Western blot analyses of S-phase-specific cyclins and p53 also support the possibility that polycystin-1 participates in regulating the progression of the cell cycle. On the basis of these observations, we believe that the PKD1 gene product, polycystin-1, might regulate $\mathrm{G} 1 / \mathrm{S}$-phase transition of the cell cycle progression.

Polycystin-1 might act as a G1 checkpoint, which controls entry into the $S$ phase, and prevents the replication of damaged DNAs caused by genotoxic reagents including UV light. Our results show that the activity of polycystin-1 is associated with p53. Previously, it was reported that polycystin-1 regulates the expression of p21. ${ }^{29}$ Hence, we think that polycystin-1 may regulate p53 and p21, the p53 downstream effector. This regulation is disrupted by the loss of polycystin- 1 activity and premature G1/S-phase transition occurs. The premature a

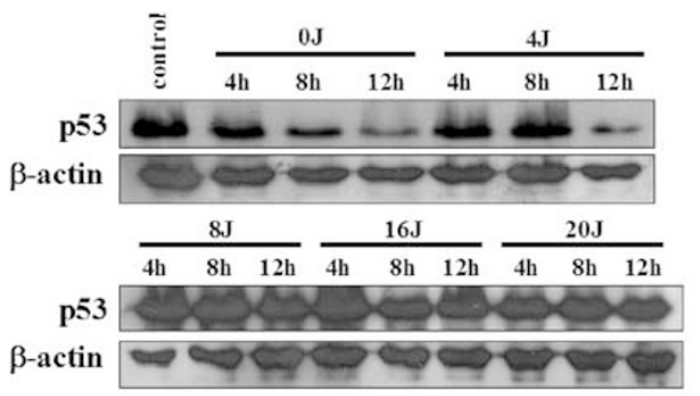

b

\begin{tabular}{|c|c|c|c|c|c|c|c|c|c|c|}
\hline b & \multirow{3}{*}{$\begin{array}{r}\text { UV dose: } \\
\text { Time: } \\
\text { No ODN: }\end{array}$} & \multicolumn{9}{|c|}{$16 \mathrm{~J}$} \\
\hline & & & $4 h$ & & & $8 \mathrm{~h}$ & & & $2 \mathrm{~h}$ & \\
\hline & & + & - & - & + & - & - & + & - & \\
\hline & 1 sense ODN: & - & + & - & - & + & - & - & + & \\
\hline & antisense ODN: & - & - & + & - & - & + & - & - & \\
\hline
\end{tabular}

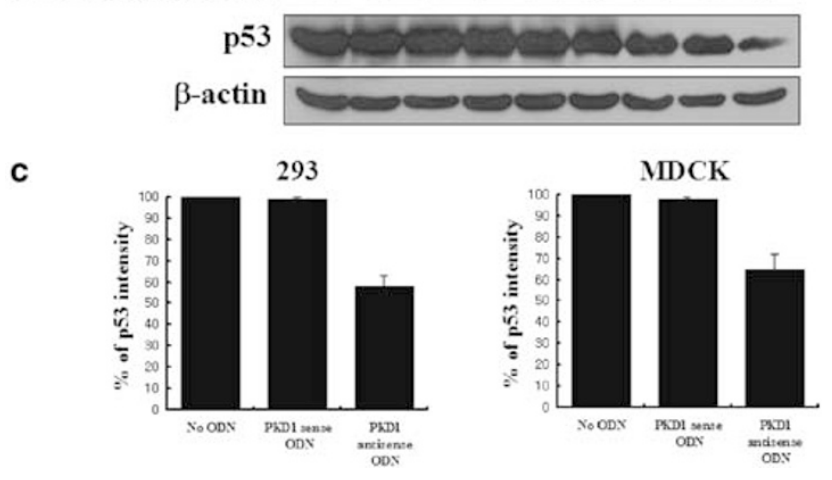

Figure 7 (a) Analysis of UV-induced p53 expression in 293 cells. 293 cells were maintained at confluence to obtain G1-phase cell populations. The cells were then diluted, treated with increasing doses of UV radiation $(0,4$, $8,16,20 \mathrm{~J} / \mathrm{m}^{2}$ ) and then were examined at the indicated times after irradiation by Western blot analysis with antip53 (DO-7: Novocastra Laboratories Ltd.). (b) 293 cells were incubated for $16 \mathrm{~h}$ after transfection with no ODN or ODNs and exposed by a UV dose of $16 \mathrm{~J} / \mathrm{m}^{2}$, and p53 was then determined. (c) The level of p53 at $12 \mathrm{~h}$ was quantified with TINA2.0. ${ }^{24}$ The level of p53 in MDCK cells was gained by Western blot analysis with anti-p53 (sc6243: Santa Cruz Biotechnology). Experiments were repeated three times and similar results were obtained.

transition might cause an accumulation of unrepaired DNA damages, which might be related to the etiology of ADPKD. However, the overall role of polycystin- 1 in the cell cycle is still unclear. More studies are required to understand the mechanisms by which polycystin-1 controls G1/S-phase transition.

\section{Acknowledgements}

This work was supported in part by a grant from the Ministry of Health \& Welfare, Republic of Korea and by a Korea University Grant. 


\section{References}

1 Striker GE, Striker LJ: Renal cysts in polycystic kidney disease. Am I Nephrol 1986; 6: 161-164.

2 Lieske JC, Toback FG: Autosomal dominant polycystic kidney disease. J Am Soc Nephrol 1993; 3: 1442-1450.

3 Ye M, Grantham JJ: The secretion of fluid by renal cysts from patients with autosomal dominant polycystic kidney disease. $\mathrm{N}$ Engl J Med 1993; 329: 310-313.

4 Grantham JJ: Polycystic kidney disease: a predominance of giant nephrons. Am J Physiol 1983; 244: F3-F10.

5 Everson GT: Hepatic cysts in autosomal dominant polycystic kidney disease. Am J Kidney Dis 1993; 22: 520-525.

6 European Polycystic Kidney Disease Consortium: The polycystic kidney disease 1 gene encodes a $14 \mathrm{~kb}$ transcript and lies within a duplicated region on chromosome 16. Cell 1994; 77: 881-894.

7 Hughes J, Ward CJ, Peral B et al: The polycystic kidney disease 1 (PKD1) gene encodes a novel protein with multiple cell recognition domains. Nat Genet 1995; 10: 151-160.

8 Bae Y, Kim H, Namgung H et al: Characterization of microsatellite markers adjacent to AP-4 on chromosome 16p13.3. Mol Cell Probes 2001; 15: 313-315.

9 Kim H, Namgung H, Kim S, Ahn C, Kang S: Development of microsatellite markers for diagnosis of autosomal dominant polycystic kidney disease. Korean J Nephrol 2000; 19: 674-680.

10 Kimberling WJ, Kumar S, Gabow PA et al: Autosomal dominant polycystic kidney disease: localization of the second gene to chromosome 4q13-q23. Genomics 1993; 18: 467-472.

11 Daoust MC, Reynolds DM, Bichet DG, Somlo S: Evidence for a third genetic locus for autosomal dominant polycystic kidney disease. Genomics 1995; 25: 733-736.

12 Ariza M, Alvarez V, Marin R et al: A family with a milder form of adult dominant polycystic kidney disease not linked to the PKD1 (16p) or PKD2 (4q) genes. J Med Genet 1997; 34: 587-589.

13 Constantinou-Deltas CD, Papageorgiou E, Boteva K et al: Genetic heterogeneity in adult dominant polycystic kidney disease in Cypriot families. Hum Genet 1995; 95: 416-423.

14 Ravine D, Walker RG, Gibson RN et al: Phenotype and genotype heterogeneity in autosomal dominant polycystic kidney disease. Lancet 1992; 340: 1330-1333.

15 Peters DJ, Spruit L, Klingel R et al: Adult, fetal, and polycystic kidney expression of polycystin, the polycystic kidney disease-1 gene product. Lab Invest 1996; 75: 221-230.

16 Bycroft M, Bateman A, Clarke J et al: The structure of a PKD domain from polycystin-1: implications for polycystic kidney disease. EMBO J 1999; 18: 297-305.
17 Carone FA, Bacallao R, Kanwar Y: Role of the matrix in autosomal dominant polycystic kidney disease. Ren Fail 1998; 20: 181-189.

18 Wagner RW: Gene inhibition using antisense oligodeoxynucleotides. Nature 1994; 372: 333-335.

19 Lin SB, Wu LC, Huang SL et al: In vitro and in vivo suppression of growth of rat liver epithelial tumor cells by antisense oligonucleotide against protein kinase C-alpha. J Hepatol 2000; 33: 601-608.

20 Ferguson PJ, DeMoor JM, Vincent MD, Koropatnick J: Antisenseinduced down-regulation of thymidylate synthase and enhanced cytotoxicity of 5-FUdR in 5-FUdR-resistant HeLa cells. $\mathrm{Br} J$ Pharmacol 2001; 134: 1437-1446.

21 Kronenwett R, Steidl U, Kirsch M et al: Oligodeoxyribonucleotide uptake in primary human hematopoietic cells is enhanced by cationic lipids and depends on the hematopoietic cell subset. Blood 1998; 91: 852-862.

22 Grantham JJ: The etiology, pathogenesis, and treatment of autosomal dominant polycystic kidney disease: recent advances. Am J Kidney Dis 1996; 28: 788-803.

23 Hong S, Kim S, Ka S, Choi I, Kang S: USP7, a ubiquitin-specific protease, interacts with Ataxin-1, the SCA1 gene product. Mol Cell Neurosci 2002; 20: 298-306.

24 Kim S, Kim T, Kim I, Hong S, Rhim H, Kang S: Polyglutamineexpanded ataxin- 1 recruits $\mathrm{Cu} / \mathrm{Zn}$-superoxide dismutase into the nucleus of HeLa cells. Biochem Biophys Res Commun 2003; 307: 660-665.

25 Peters DJ, van de Wal A, Spruit L et al: Cellular localization and tissue distribution of polycystin-1. J Pathol 1999; 188: 439-446.

26 Ibraghimov-Beskrovnaya O, Bukanov NO, Donohue LC et al: Strong homophilic interactions of the Ig-like domains of polycystin-1, the protein product of an autosomal dominant polycystic kidney disease gene, PKD1. Hum Mol Genet 2000; 9: $1641-1649$.

27 Griffin MD, Torres VE, Grande JP, Kumar R: Immunolocalization of polycystin in human tissues and cultured cells. Proc Assoc Am Physicians 1996; 108: 185-197.

28 Rory KG, Hatsumi N, John BL, Carl GM: Role and regulation of p53 during an ultraviolet radiation induced G1 cell cycle arrest. Cell Growth Differ 2000; 11: 149-156.

29 Bhunia AK, Piontek K, Boletta A et al: PKD1 induces p21(waf1) and regulation of the cell cycle via direct activation of the JAKSTAT signaling pathway in a process requiring PKD2. Cell 2002; 109: $157-168$. 